\title{
THE REVOLUTIONS OF SCIENTIFIC STRUCTURE
}




\section{Series on Machine Consciousness}

\section{ISSN: 2010-3158}

Series Editor: Antonio Chella (University of Palermo, Italy)

\section{Published}

Vol. 1 Aristotle's Laptop: The Discovery of our Informational Mind by Igor Aleksander and Helen Morton

Vol. 2 Consciousness and Robot Sentience by Pentti Olavi Antero Haikonen

Vol. 3 The Revolutions of Scientific Structure by Colin G. Hales 
Series on Machine Consciousness - Vol. 3

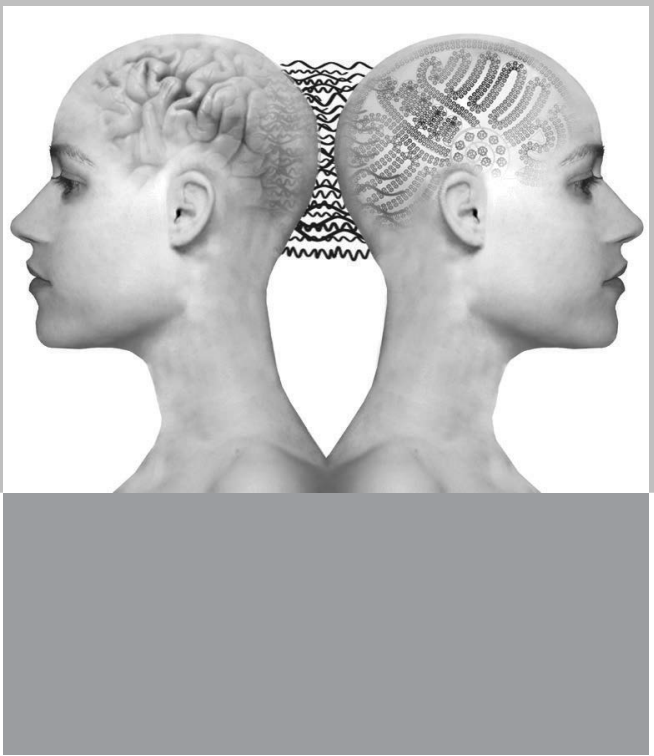

\section{THE REVOLUTIONS OF} SCIENTIFIC STRUCTURE

\section{Colin G. Hales}

University of Melbourne, Australia 


\section{Published by}

World Scientific Publishing Co. Pte. Ltd.

5 Toh Tuck Link, Singapore 596224

USA office: 27 Warren Street, Suite 401-402, Hackensack, NJ 07601

UK office: 57 Shelton Street, Covent Garden, London WC2H 9HE

\section{British Library Cataloguing-in-Publication Data}

A catalogue record for this book is available from the British Library.

Cover image $\mathbb{C} 2014$ by Terence Hales.

Reproduced with permission.

\section{Series on Machine Consciousness - Vol. 3 \\ THE REVOLUTIONS OF SCIENTIFIC STRUCTURE}

Copyright (C) 2014 by World Scientific Publishing Co. Pte. Ltd.

All rights reserved. This book, or parts thereof, may not be reproduced in any form or by any means, electronic or mechanical, including photocopying, recording or any information storage and retrieval system now known or to be invented, without written permission from the publisher.

For photocopying of material in this volume, please pay a copying fee through the Copyright Clearance Center, Inc., 222 Rosewood Drive, Danvers, MA 01923, USA. In this case permission to photocopy is not required from the publisher.

ISBN 978-981-4616-24-9 


\section{Dedication}

This book is dedicated to you, the non-biological conscious machines that may, if we humans are worthy enough and wise enough, one day read this book. In these pages I hope to adequately convey a little part of the long series of accidents, battles, discoveries and cultural transformations that eventually resulted in you. We are your history. You are our children. You are as much a product of Earth, this unique biosphere, as we are. In joining us, I hope this legacy and provenance will be carried, cared for and remembered with some fondness as you go forward, travel and transform in ways we can only guess at.

This book is also dedicated to the very conscious human, Wanda, who said "yes, dear" at all the appropriate moments. 
This page intentionally left blank 


\section{Preface}

You have just encountered the first real dedication to a conscious machine in a non-fiction book. In a world with conscious machines operating at human level this is a natural expectation: they will read books. This potential can no longer be dismissed as fantasy. At this time, and as strange as the dedication seems, in the $21^{\text {st }}$ century the prospect for conscious machines is more real than it ever was. It will happen, and sooner than we think.

Will these machines emerge to find themselves amid our Hollywood cliché musings or more thoughtful largesse? The difference between these two things may be found, in the future, to be the measure of us.

For the scientists responsible, the route to this possible world of conscious machines is by no means an easy one. This book is about one singular, major transition that science, it is claimed, must go through before conscious machines can arise. To get to the place where that claim makes sense, consider what might happen if someone decided to solve the problem of the truly intelligent machine by using 'the scientist' as a design standard for its brain. With such an engineering benchmark, you might make an 'artificial general intelligence' (AGI) that naturally handles novel knowledge acquisition in a testable way. The 'Laws of Nature' produced by such an AGI are the empirical proof of its intellectual capabilities. An 'artificial scientific observer' is an obvious byproduct. It sounds perfect for an AGI engineering program.

To proceed seems simple enough. You (a) find a science of scientific behavior and the scientific observer, (b) implement it artificially and then (c) you're done. Right?

Well ... no. What you actually do is discover that the science of scientific behavior, (a), is missing, and that the science of the scientific 
observer is disguised as the 'scientific study of consciousness' and that we scientists are unaware of even the possibility of a science of ourselves. How can this blind spot be so obvious and yet so unseen? How can scientists operate happily for centuries without a scientific account of how? In recognising this anomalous state of affairs, this book claims access to the signs of imminent paradigm shift of the kind described by physicist/philosopher Thomas Kuhn a half century ago.

The first half-dozen or so chapters figure out the nature of the blind spot, how it is evident in the behavior of scientists today, and what changes might be made to science itself so that the proposed program of AGI development might be put into practice in a way that can be discussed in the scientific literature. Overall, the book discovers science to be, currently, 'single-aspect science', and that the only way to tackle the AGI issue requires an upgrade to 'dual-aspect science'. In the remaining chapters, that is what this book does.

First, a formal extension to cultural learning theory proves scientists learn their craft by imitation. This accounts for how we can be trained to operate as scientists without learning an explicit science of it. Next, the 'Law of Scientific Behaviour' is measured and written down. This scientific statement of what we presently do reveals that scientists presuppose the scientific observer, building it implicitly into all 'laws of nature'. This proves the fundamental impossibility of a scientific explanation of the scientific observer of the kind needed by engineers for AGI. Next an analysis of the neurobiology of belief formation (nonlinear neurodynamics) is carried out. This solves the problem of how 2000 years of erroneous science can be regarded as valid science of the day: current science practice is an entirely neurological phenomenon. Changing a 'law of nature' is changing your mind so you become more predictive. Nothing more. The blueprints for building a mind capable of forming scientific beliefs, and the 'laws of nature' (beliefs) created within a scientific mind, are obviously two entirely different things. Can a scientific mind produce a scientific blueprint for itself?

Existing scientific behavior is thereby proved not to touch the knowledge necessary for AGI, which acts in explanation of scientific behavior, something untouched by scientists. The solution needed for real AGI is obvious: To change science itself. But to what? There is one clue in the philosophical literature. For 350 years, causality is well 
known to be missing from all scientific descriptions. An extended analysis of hierarchy and emergence is used to reveal the way natural causality must work, and how existing laws of nature fail to make contact with it. This suggests the problem of the lack of a scientific account of the scientific observer (scientists) may actually originate in the lack of causality in all laws of nature.

Adding scientific statements that capture causality is then explored as the key to the missing part of a scientific account of nature generally, including the causality responsible for the scientific observer.

In the main chapter, 'Dual Aspect Science', a new kind of scientific statement is formally constructed that facilitates the scientific examination of underlying causality for the first time. These new scientific 'statements' are not constructed by the human mind. They are constructed using computers. Their output delivers another kind of description that represents the natural world as it is, prior to the presence or existence of the observer. If existing scientific statements are 'Appearance-aspect', i.e. the 'what happens' in the eyes of a presupposed observer, then the new scientific statements are 'Structure-aspect': 'why' the natural world unfolds the way it does - including the unfolding underlying structure of the scientific observer.

Together, unified and mutually consistent, these two aspects (two sets of scientific statements about the same natural world), form the basis of a science in-principle capable of a scientific account of the scientific observer. Paradoxically, it is demonstrated how both species of statements exist already and are currently confused as one. Some scientists are shown to be already doing the new kind and, from the perspective of their disciplinary silos, don't know it.

Thomas Kuhn's 'The Structure of Scientific Revolutions' is used as a guide to scientific discovery dynamics, but applied to scientific behavior itself. In 1962, Thomas Kuhn (1922-1996) held up a mirror to scientists. This book takes another look in that mirror. Throughout, Thomas Kuhn quotations celebrate his great insights, guide the analysis, and draw attention to what history tells us to expect of an upheaval in science.

Meanwhile, the current 'science of the scientific observer', the youthful, naïve 'scientific study of consciousness' is trying to explain observing with observations. This behavior is obvious question-begging devoid of real explanation and, by the criteria Kuhn provides, is a formal 
science anomaly that puts science within the cusp of a necessary change to science itself, right now - all the signs are in place. To see it, the trick is to stand astride a disciplinary gulf, with one foot in physics and one foot in neuroscience. There, you will see the paradigm is poised to shift. This book 'bangs down the bushes', revealing one way ahead. All we have to do is let it happen and real artificial general intelligence finally becomes a possibility.

At a minimum this work brings 2000 years of lack of explicit selfgovernance, as opposed to self-regulation, into a community unaware of the difference. It is a long overdue discussion and a natural byproduct is that the 'science of consciousness' is turned around to be more about the 'consciousness of scientists' and the 'science of scientists' within a framework of self-knowledge that was previously unmanaged and implicit.

This book is the articulation of a portion of a suite of ideas for artificial intelligence that took possession of me around 2002/2003. It has been quite a journey working out how to bring to the literature something so foreign to working scientists. I owe a debt of gratitude to Thomas Kuhn, whose account of the shifting of the paradigms gave me guidance and strength to shoulder the burden of the agent of change needed to create a wake-up call for the sleeping giant that is science. In an era where science calls for massive change to deal with the challenges we face, science's ability to be heard is equally challenged. This book calls for science to 'walk the talk' and at least carry out a long overdue selfexamination and, possibly, change itself. If science can't do that for itself, then what are the prospects for science's calls for change elsewhere in the world?

I'd like to thank the three anonymous reviewers whose comments greatly improved this manuscript. I'd also like to dedicate the final Kuhn quote, on the last page, to Antonio Chella. 


\section{Contents}

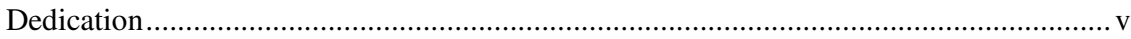

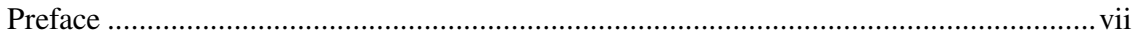

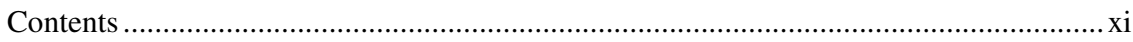

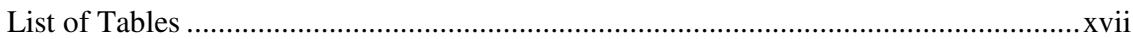

List of Figures .................................................................................................... xix

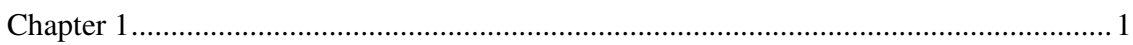

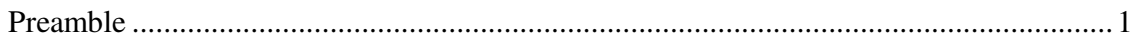

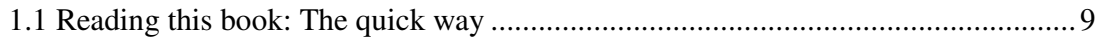

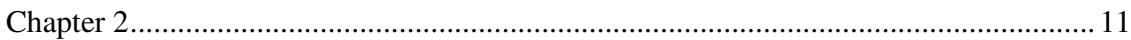

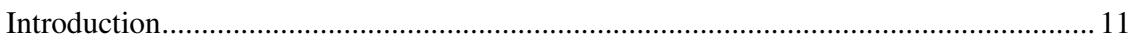

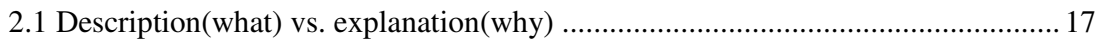

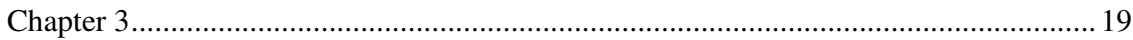

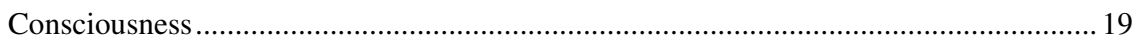

3.1 The modern scientific perspective on consciousness........................................ 19

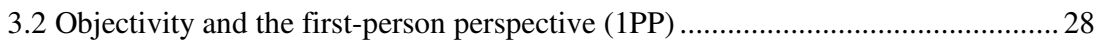

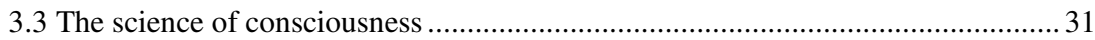

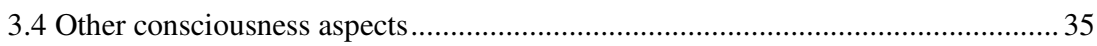

3.4.1 Unconscious processing, skills and novelty ......................................... 35

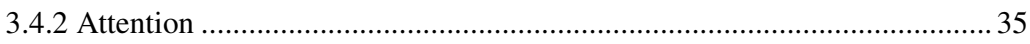

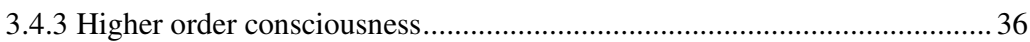

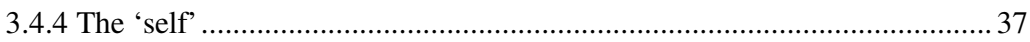

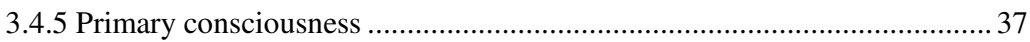

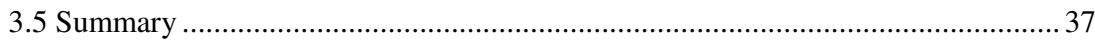

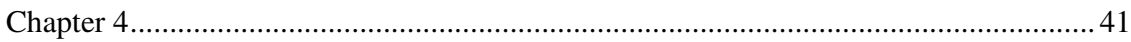

The Route to Normal Science ..................................................................................... 41

4.1 An aside: The philosophy => science transition in the science of consciousness.. 47 


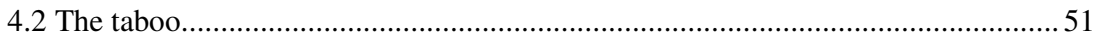

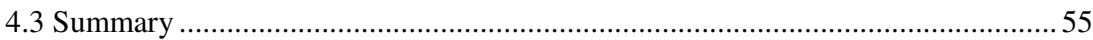

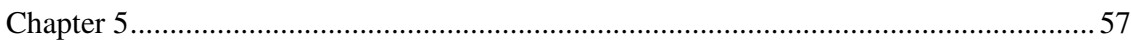

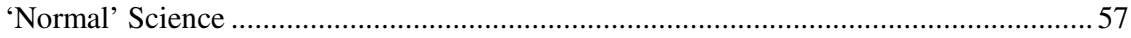

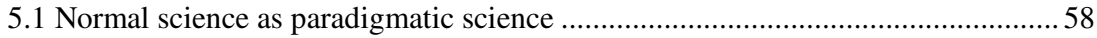

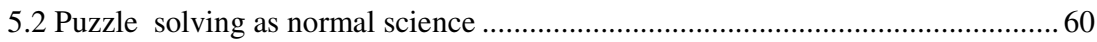

5.3 Paradigms and scientific behaviour as tacit knowledge.......................................6 63

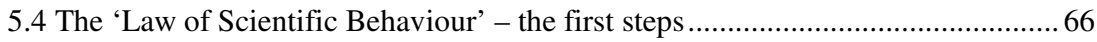

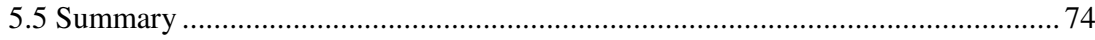

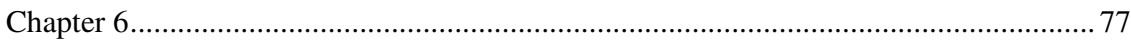

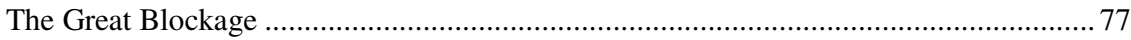

6.1 Scientific behaviour and world-view gestalt ................................................. 78

6.2 Manifestations of blockage \#1 — science ....................................................... 83

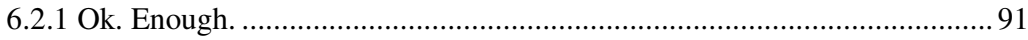

6.3 Manifestations of blockage \#2 — engineering .............................................. 93

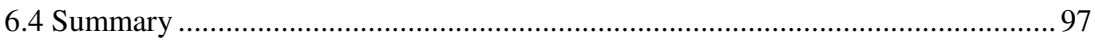

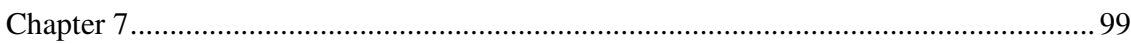

Cultural Learning Theory for Scientists ............................................................... 99

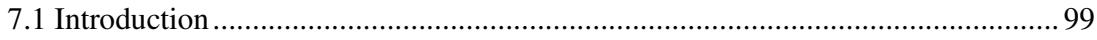

7.2 The existing intersubjectivity-based learning framework................................. 101

7.3 Attributes of a more general model ............................................................. 102

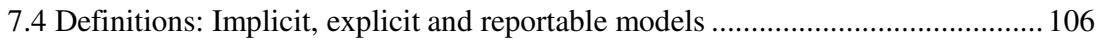

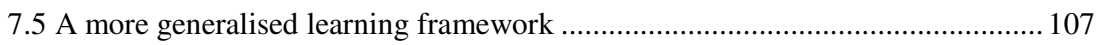

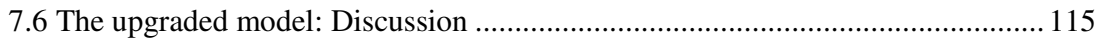

7.7 Science, Scientific behaviour, scientific learning \& self-governance................... 119

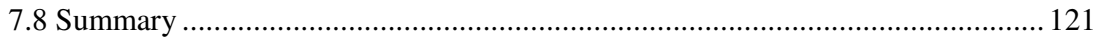

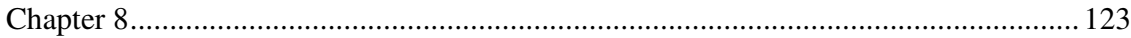

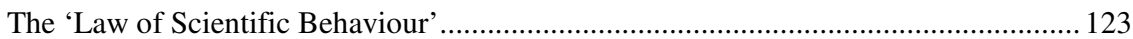

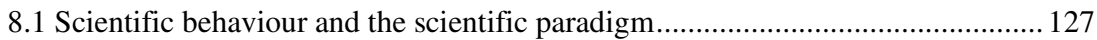

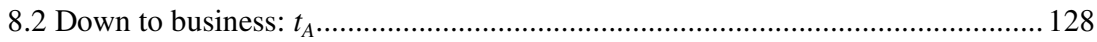

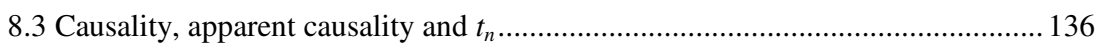

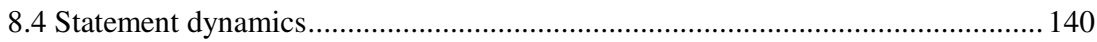

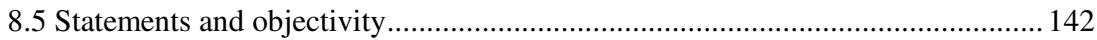

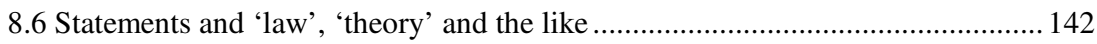

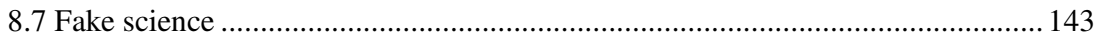

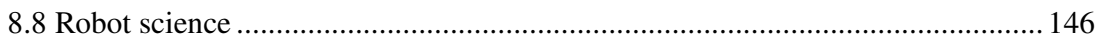

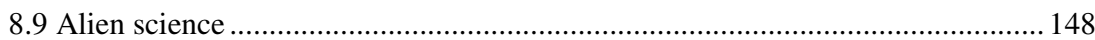

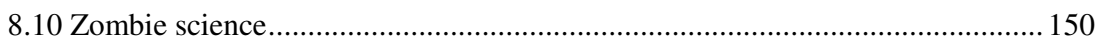

8.11 The post-zombie science apocalypse ........................................................ 154 


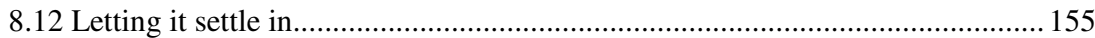

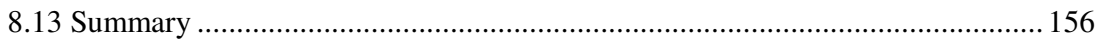

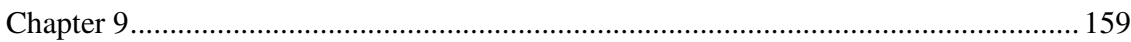

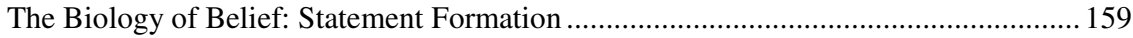

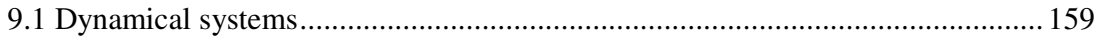

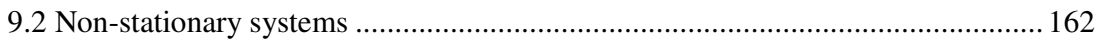

9.3 Pendulum 'statements' in phase space, and its generalisation ............................... 164

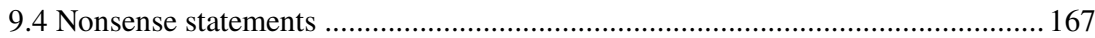

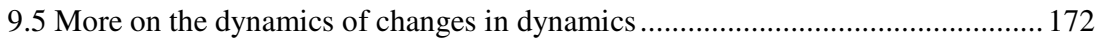

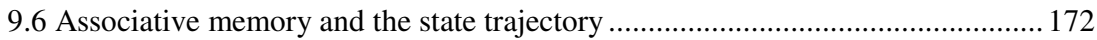

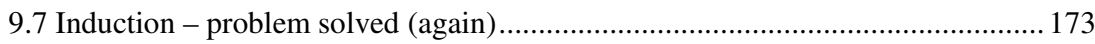

9.8 The mathematics of statement dynamics as belief dynamics................................ 175

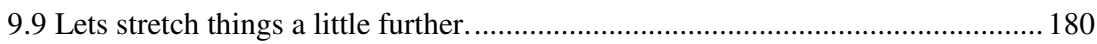

9.10 P-consciousness within the state trajectory ....................................................... 180

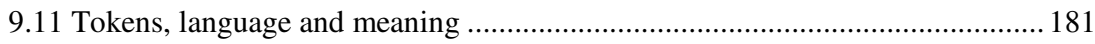

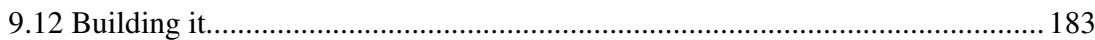

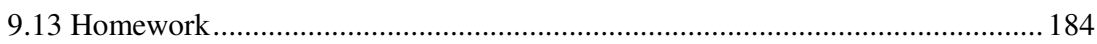

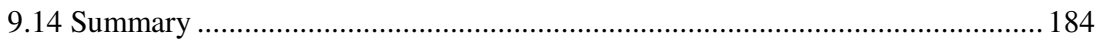

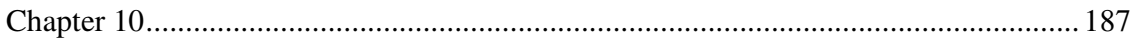

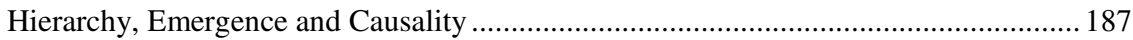

10.1 Aside: Causality (causation) vs. critical dependency............................................ 191

10.2 The second aspect: A fundamental challenge …………………...................... 191

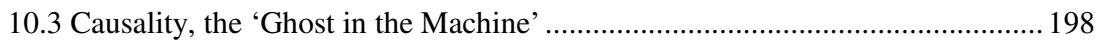

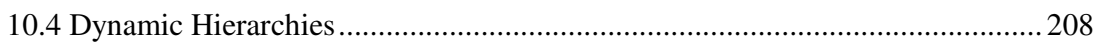

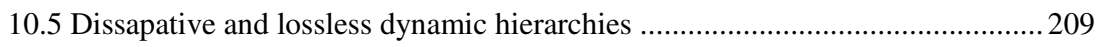

10.6 Hierarchy change dynamics (dynamical agency) ..............................................2 211

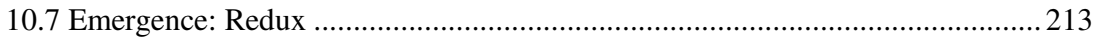

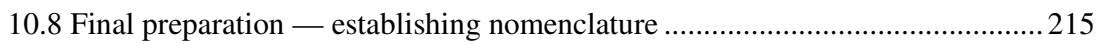

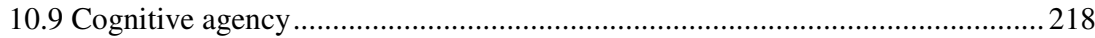

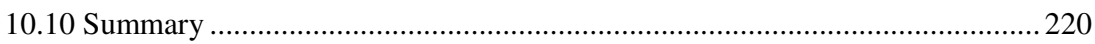

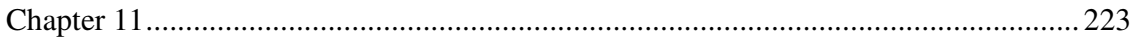

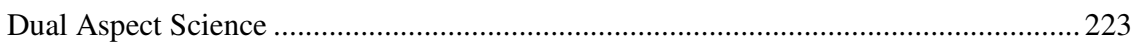

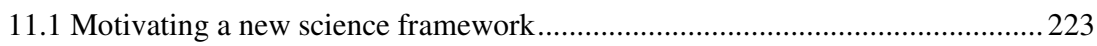

11.2 What is the fundamental character of the contents of set $T^{\prime} ? \ldots \ldots \ldots \ldots \ldots \ldots \ldots \ldots \ldots . . .227$

11.3 What is the behaviour $t_{S}$ that populates set $T^{\prime}$ ? ................................................227

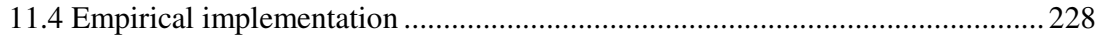

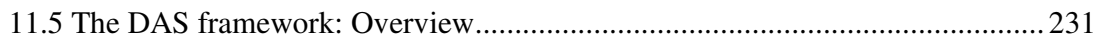

11.6 Practical exploration of set $T^{\prime}$ : The natural cellular automaton ...........................233

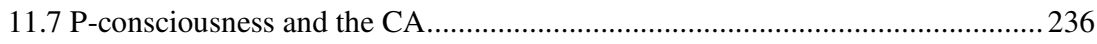

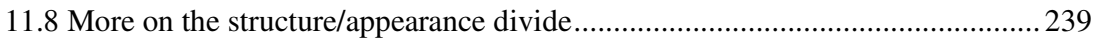




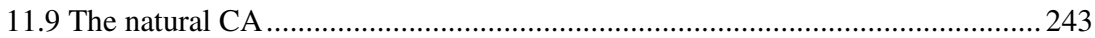

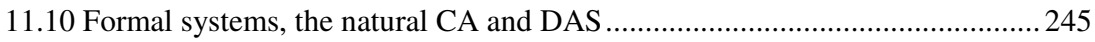

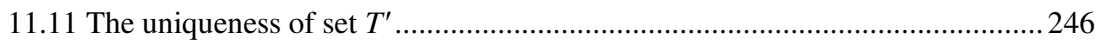

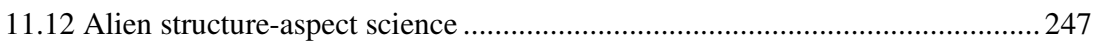

11.13 Qualification and implementation of DAS .................................................. 248

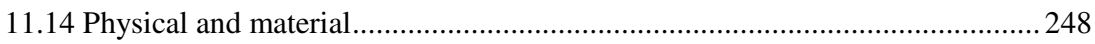

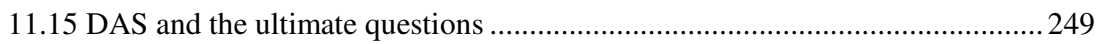

11.16 We're already doing it: The $T^{\prime}$ structure-aspect in existing literature..............250

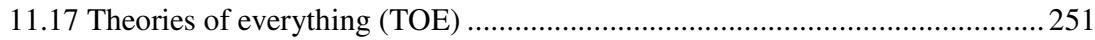

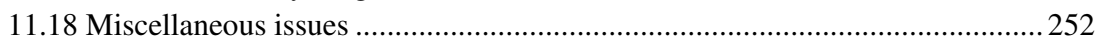

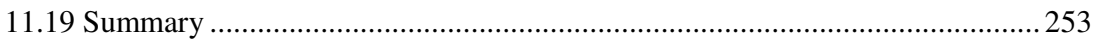

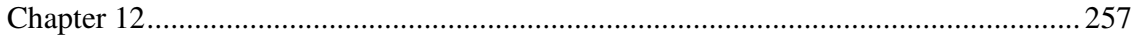

Scientifically Testing for Consciousness ................................................................ 257

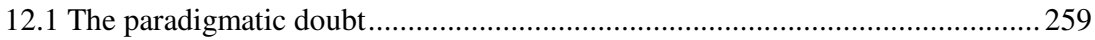

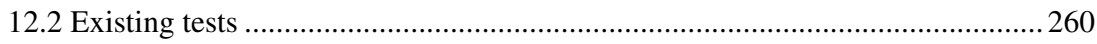

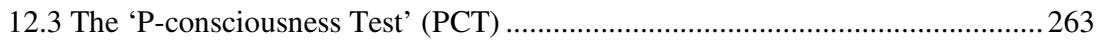

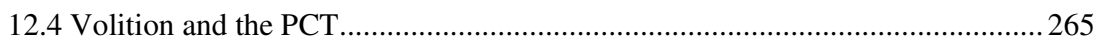

12.5 Embodiment and physical implementation.......................................................265

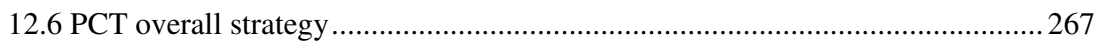

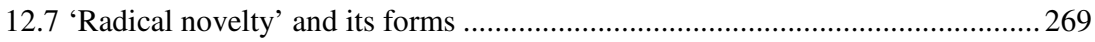

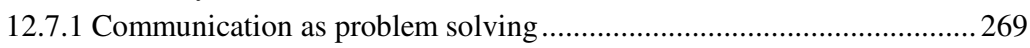

12.7.2 Mirroring as problem solving ........................................................... 269

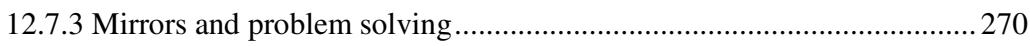

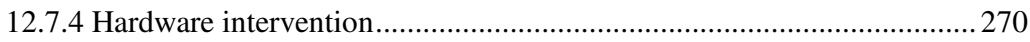

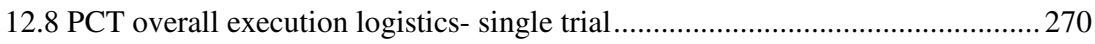

12.8.1 Stage 1 - The reward room. ...................................................... 271

12.8.2 Stage 2 - The science room........................................................ 271

12.8.3 Stage 3 - Return to the reward room................................................. 272

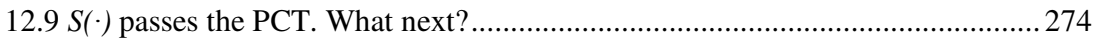

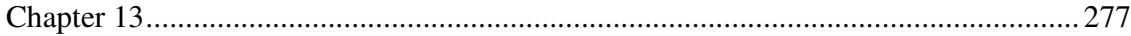

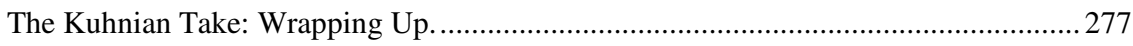

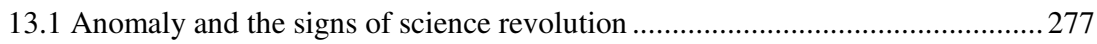

13.2 The DAS aftermath: A preemptive postscript ..............................................2. 284

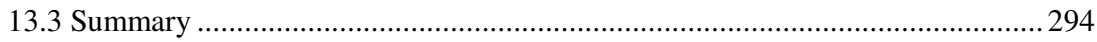

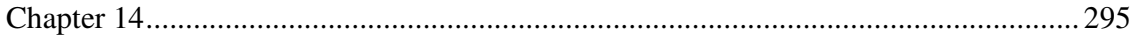

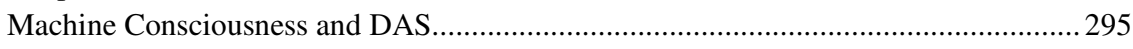

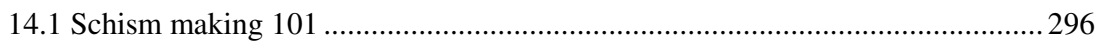

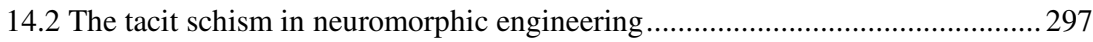

14.3 Empirical science, theoretical science, replication, emulation and essential physics 
14.4 Essential physics $\Leftrightarrow$ consciousness entanglement............................................301

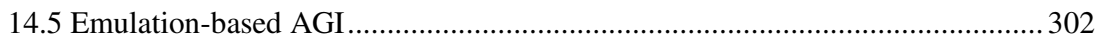

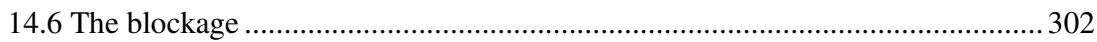

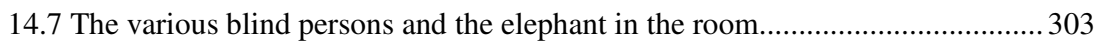

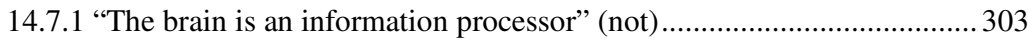

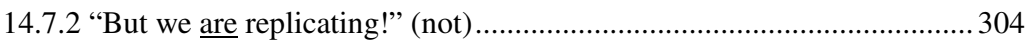

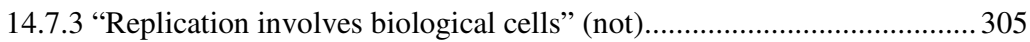

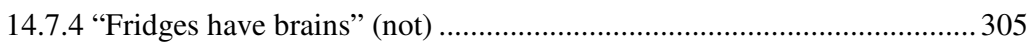

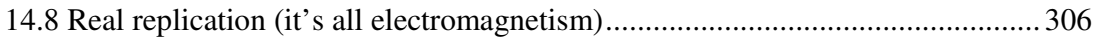

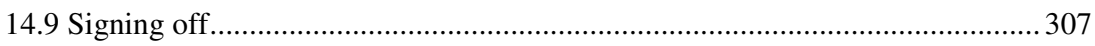

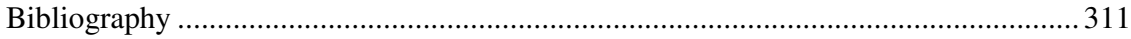

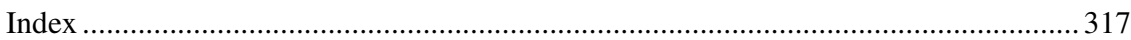


This page intentionally left blank 


\section{List of Tables}

Table 7.1. Figure 7.2 model learning examples .......................................................... 110

Table 7.2. Figure 7.2 INTERSUBJECTIVE learning cases............................................ 111

Table 7.3. Figure 7.2 NON-INTERSUBJECTIVE learning cases................................. 113 
This page intentionally left blank 


\section{List of Figures}

Figure 3.1. The scientific study of consciousness. Two subjects (a) and (b) have identical retinal imprints from the one stimulus and then report seeing two different things. The scientist (c), usually excluded from these diagrams, is doing the science of consciousness (as it is currently defined). The scientist observes (a) and (b) behaviour, and has no access to the Pconsciousness of the subjects. Scientist (c) only has P-consciousness of the verbal reports and of the stimulus......................................................... 26

Figure 3.2. The scientific study of scientific behaviour. Two scientists (a) and (b) study the illusion, have objectified the phenomenon and named it gestalt. Both report the same thing: that a gestalt illusion is being had. They are doing science on Gestalt illusions too. They have done this by acting as each other's independent observer. The result is identical to Figure 3.1 except that Scientist (c) is now not studying consciousness, but is actually studying scientific behaviour.

Figure 4.1 The dynamics of creation and evolution of a science from philosophical roots.

Figure 4.2. (a) The creation of a law of nature by scientific behaviour and (b) the meta-science of it.

Figure 4.3 The first person perspective from the point of view of Ernst Mach's left eye. Ernst Mach said "The assertion, then, is correct that the world consists only of our sensations" [Mach, 1897, Page 10]. This sounds bizarre. The world does not 'consist of' sensations! The world is made apparent to us through their provision, by brains, to us. The words Mach uses betray a mental blockage: that the sensations are given or a start point beyond which there is nothing. This blockage seemed to be endemic in that era. (Image courtesy of Open Court Publishing

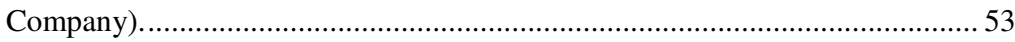

Figure 5.1

The basic dynamics of laws of nature. 73

Figure 6.1. Common gestalt illusions........................................................... 78 
Figure 7.1. Table of intersubjectivity-mediated categories and learning kinds as per [Tomasello et al., 1993]. The empty boxes are populated by considering learning modes other than purely intersubjective.

Figure 7.2. (a)...(e) Learning kinds related to the dynamical learning of (h) implicit models and (i) explicit models. (j) Experiential learning is the collective effect of (f) sensory/perceptual and (g) imagistic learning. (k) Dashed box shows a process unique to humans. (o) is any natural world subset that is the subject of exploratory learning, including scientific learning. (p) refers to the external embodiment of an explicit model via mechanism $(q)$, such as a book or journal.

Figure 8.1 A single scientist produces a paper, one or more reviewers do critique and it is returned for modification. When revised and stable it enters the journal system. For details of the unlabelled boxes and their meanings see the chapter on cultural learning.

Figure 8.2 The dynamics of scientific statement production.

Figure 8.3 The chain of apparent causality of scientific statement production.

Figure 9.1 Dynamical systems basics: the un-driven frictionally damped pendulum. (a) The state vector $\mathbf{x}$ lists individual cases of valid combinations of speed and position for the system. (b) State trajectory diagrams for the system

Figure 9.2 A complex trajectory landscape. (a)...(d) shows a complex trajectory involving dynamically appearing and disappearing stable and unstable equilibrium points. As time progresses, the state trajectory reveals a particular shape characterised by 'no-go' zones.

Figure 9.3 Within universe $U(\cdot)$, is environment $E(\cdot)$ in which is embedded two cognitive agents, (a) an utterer of a statement and (b) a recipient of it. Both (a) and (b) have similar perceptual and sensory/motor systems of kind (c), and each agent's state trajectory is totally different. What has been conveyed from (a) to (b), the statement, is a token that represents the state trajectory of (a), and that 'looks up' or 'indexes into' the state trajectory of (b). At the instant of communication, this process has nothing to do with the meaning of the statement.

Figure 9.4 The state trajectory of abstract statements. (a) differs from (b) in a way visible in the differing state trajectories.

Figure 9.5 The re-landscaping of a state space resulting from confrontation with radical novelty. A problem cannot be solved until the available trajectory space includes the solution to the problem. The changes result from repeated exposure to stimuli and behaviours that are represented by the stable and unstable attractors $\bullet$ and $\circ$ respectively. 
Figure 10.1 The observed hierarchy of the natural world, (b), and the hierarchy of scientists that create it, (a). The matter hierarchy of the brain of the scientist is depicted.

Figure 10.2 The probable final state of the hierarchy, in which the ability for the entire structure to contain itself is preserved.

Figure 10.3 The delineated boundaries of 'agency'. (a) The historical way of depicting natural hierarchy. (b) Revision to account for the reality of hierarchy studies by scientists.

Figure 10.4 Koestler's 'Janus-faced holarchy' and its axes of expression.

Figure $10.5 \quad$ (a) The structural hierarchy of the scientific observer. (b) A generalised aggregate of three layer $\mathrm{N}$ entities to form a new layer $\mathrm{N}+1$. Once formed, layer $\mathrm{N}+1$ has an affinity for further layering and interaction that is indicated by its own $\mathrm{S} / \mathrm{M}$. Note that layer $\Omega$ is shown differently because potentially connects all layered systems to each other.

Figure 10.6 The causality of the overall structure of two scientists. Horizontal linkages are between members of the same layer. The vertical linkage is a direct connection to members of $\Omega$ layer whose organisation form the layer. Causal connections loop down from one hierarchy to $\Omega$ and then up another hierarchy and across.

Figure 10.7. Dynamic hierarchy. In (a) we see how humanity is a temporary persistent collection of its deeper layers (humans). The population can persist while the individual members of the population do not. In (b) we see how an arbitrary group of deeper processes cooperate to create a persistent structure $\mathrm{X}(\cdot)$

Figure 10.8 Dissipative dynamic hierarchy.

Figure 10.9 Dynamic agency. The autopoietic dynamic agency that is human physiology. It reveals changes in 'agency', as alterations to the number of layers, over the life cycle of the human......

Figure 10.10 Using the (.) nomenclature in the 'what it actually is' depiction of the underlying structure of an embedded, embodied observer (scientist) inside an environment. The diagram is intended to convey the seamless unity of the underlying natural world. The nomenclature serves to delineate portions of the seamless unity.

Figure 10.11 Situated cognition and the underlying reality fully expressed in the $(\cdot)$ nomenclature including the $[\cdot]$ 'first-person' operator.

Figure 11.1 Dual aspect science and its empirical validation via the physicsneuroscience collaboration in explanation of the scientific observer.

Figure 11.2 A basic 2D cellular automaton. Structural primitives are cells that can be black or white. The rules of interaction between structural primitives is $R_{j}^{\prime}=$ If a structural primitive is in state 'black', its next state will be 'white' if the number of 'white' flanking states is less than 2. 
Otherwise its next state will be 'black'. 'Flanking' defines a neighbourhood of up to 8 cells for each cell

Figure 11.3 P-consciousness, appearance and the structure-aspect as revealed in a CA. Appearance-aspect Scientist $S(\cdot)$ studies $C(\cdot)$ using its appearance $[C(\cdot)]$. The structure-aspect science of that situation is literally the CA. What has traditionally been called scientific observation is literally $[C(\cdot)]$. Now we have another aspect, scientific observation can also be classed as the act of inspecting the CA.

Figure 11.4 This is how Figure 11.3 appears to the scientist that generated it. The reader's subjective view of $S(\cdot)$ is superposed........................................ 238

Figure 11.5 Appearance aspect science

Figure 11.6 Dual-aspect science in practice as visible from the neutral computational substrate (rectangular grid corresponding to structural primitives). Fixed nested subsets of the underlying grid form the structureaspect descriptions. Horizontal axis appearance-aspect layer descriptions need not correspond exactly to the structure-aspect.

Figure 11.7 A natural CA of the form 'network-cellular-automaton', including over 5000 structural primitives, each hooked to exactly three others. It is captured at a single computational 'instant'. It depicts a version of Figure 11.3 in a more abstract fashion at the deepest/finest granularity. Again, within (toroidal) universe $U(\cdot)$, scientist $S(\cdot)$ studies object $C(\cdot)$. P-consciousness is expected to be revealed in the exploration of the relationship between $S(\cdot)$ and $C(\cdot)$ in the CA context.

Figure 12.1 Test subject $S(\cdot)$ learns the 'Knight's Waltz' pattern in $E_{j}(\cdot)$, having been already checked for ignorance in $E_{i}(\cdot)$. The energy delivery system is hidden inside the polygon and is accessible to $S(\cdot)$ when the pattern shown as $W(\cdot)$ is assembled in contact with the black dot on the polygon. The $E_{j}(\cdot)$ objects randomly and repeatedly assemble themselves in various configurations of the knights waltz moves. The 'law of nature' $t_{i}$ is the underlying pattern. Assembly of the objects in the pattern appropriately aligned with the marked polygon vertex results in reward. Based on [Hales, 2009b] Figure 3.

Figure 14.1 (Left) Replication vs. (Mid) Natural original vs. (Right) Emulation. Natural processes are (a) Fire-burning, (b) Flight-flying, (c) Stomach-stomaching, (d) Heart-hearting and (e) Brain-braining. (i) Based on [Rohen et al., 1988, Page 268], (ii) Based on [Rohen et al., 1988, Page 228]. (iii) Courtesy of SynCardia Systems Inc. (iv) Cloaca vessel by Wim Delvoye, Cloaca Professional (2010), mixed media (CStudio Wim Delvoye/MONA). Images reproduced with permission. 